Pacific Journal of Mathematics

MULTIPLICATIVE PERTURBATION OF SEMIGROUP 


\title{
MULTIPLICATIVE PERTURBATION OF SEMIGROUP GENERATORS
}

\author{
K. Gustafson AND G. Lumer
}

\begin{abstract}
Contraction semigroups of linear operators on a Banach space appear in a wide variety of physical and mathematical situations, and for this reason there has been much interest attached to studying the properties of such semigroups and their infinitesimal generators. In this paper we examine in some detail the question of when one can (left or right) multiply the infinitesimal generator $A$ by another (bounded or unbounded) operator $B$ and still preserve the generator property. Also, we state when polynomials in a generator retain the generator property. Our treatment is not exhaustive but is meant to be representative of the type of results which may be obtained.
\end{abstract}

Section 2 contains a brief background of material necessary to our presentation, including the relevant previous results; since there have been many recent developments in the theory of linear (and nonlinear) equations of evolution while this paper was in preparation, in $\S 2$ we have added a few comments pointing out some of those results as they pertain to this paper. In $\S 3$ we employ the approach that was used in Gustafson [8] for bounded left multipliers; the two principal ingredients are the index theory of (unbounded) operators and the dissipativeness of the operator products involved. The main idea here is to arrive at multiplicative perturbation results by using additive perturbation results. Another approach to the multiplicative perturbation question is to use direct semi-group methods to solve the initial value problem with generator $B A$, as was done for example in the recent paper Calvert and Gustafson [1]. In $\S 4$ we develop some other methods, give some examples, and obtain a criterion for a polynomial of a generator to be a generator. Although we do not attempt to treat specific applications per sé in this paper, let us mention at this point (see also $\$ 4$ ) the descriptive situation in which the semigroup is induced by a Markov process and the left multiplication is induced by a stochastic time change.

An implicit corollary to this paper is that after the operator theoretic considerations are taken care of, the basic requirement on the perturbed operator governing whether or not it retains the generator property is that it remain dissipative. This point was mentioned earlier in [8] as concerns bounded left multiplicative perturbation, but deserves emphasis since it pertains as well to any multiplicative, additive, or "polynomial" perturbation, and would apply as well to any 
functional calculus developed for generators. We do not here consider criteria for perturbed dissipativeness; some results in that direction may be found in $[9,10,12,17,22]$. Of course often the perturbed operator may be verified to be dissipative directly (e.g., by integration by parts), or by choice of a special semi-inner product as in Dorroh [3], by commutativity properties of $A$ and the perturbation of $A$, or by other methods motivated by the particular application involved.

The authors would like to thank the referee for several useful suggestions yielding more general statements of some of the results of $\S 4$.

2. Previous and current results. We will have need to refer to a certain background of previous results. Throughout this paper $X$ will denote an arbitrary complex Banach space, and all operators $T$ will have dense domain $D(T)$ and range $R(T)$ in $X ;[x, y]$ will denote a semi-inner product as defined by Lumer [19]. Every Banach space possesses at least one semi-inner product compatible with the norm (see $[5,19,20,21]$ ).

We will restrict our attention to contraction semigroups, although the results can be extended to bounded semigroups by translation of the infinitesimal generator. Let $G(1,0)$ denote the class of all linear operators $A$ which are the infinitesimal generators of strongly continuous (class $C_{0}$ ) semigroups of contraction operators on $X$; see [16, 18] for more details. In Lumer and Phillips [21] the following version of the Hille-Yosida Theorem characterizing $G(1,0)$ was obtained. Let $\theta(A)=\sup \operatorname{Re}[A x, x],\|x\|=1, x \in D(A) ; A$ is called dissipative if $\theta(A) \leqq 0$.

THEOREM 2A. A necessary and sufficient condition for a linear operator $A$ with dense domain to generate a strongly continuous semigroup of contraction operators is that $A$ be dissipative and that $R[I-A]=X$.

In particular, $A \in G(1,0)$ if $A$ is closed, dissipative, densely defined, and $R[\lambda-A]$ is dense for some $\lambda>0$.

In [3] Dorroh showed that when $X$ was a space of bounded functions and $A \in G(1,0)$, then $p A \in G(1,0)$ when $p$ was the operator on $X$ given by multiplication by a positive bounded function, bounded below above zero. In $[8,9]$ the following general result along these lines was obtained. An operator $B$ is called strongly accretive if there exists $m>0$ such that $\operatorname{Re}[B x, x] \geqq m,\|x\|=1, \forall x \in D(B)$; in the following statement $B$ is to be bounded and everywhere defined. 
Theorem 2B. For any Banach space $X$, let $A \in G(1,0)$ and either of the following (disjoint) conditions hold for $B$ :

(i) $B$ is strongly accretive;

(ii) $\exists \tau>0 \in \tau\left\|B^{-1}\right\|^{-1}>\|\tau B-I\| \geqq 1, \theta(-B) \geqq 0$. Then $B A \in G(1,0)$ if and only if $B A$ is dissipative.

We will use the following results concerning additive perturbation of $G(1,0)$ and preservation of operator indices which may be found in $[7,11]$.

Theorem 2C. Let $A \in G(1,0), A+B$ dissipative, $\|B x\| \leqq a\|x\|+$ $b\|A x\|$ with $b<1$ for all $x \in D(A)$. Then $A+B \in G(1,0)$.

THEOREm 2D. Let $\|B x\| \leqq a\|x\|+b\|T x\|$ for all $x \in D(T)$, where $b<1-a\left\|T^{-1}\right\|$. Then $\beta(T)=\beta(T+B)$.

Here $\beta(T)=\operatorname{dim} X \overline{R(T)}$ is the deficiency index of $T$; since in Theorem $2 \mathrm{D}$ the operators $T$ and $T+B$ are $1-1, \beta=-\kappa$, where $\kappa$ is the index of the operators; in general, $\kappa=\alpha-\beta$, where $\alpha$ is the nullity of the operator. Theorem $2 \mathrm{D}$ holds for arbitrary operators in a normed linear space [11] but for semi-Fredholm operators in a Banach space it is due to Kato (see [18]); sometimes (e.g., see [6]) $\beta(T)$ is used to denote the deficiency of $R(T)$ rather than $\overline{R(T)}$, but all of our ranges will turn out to be closed, so the distinction will vanish.

We use the term invertible to mean that an operator possesses a bounded everywhere defined inverse.

For use in $\S 4$ let us state the following lemma.

Lemma 2E. Let $T$ be dissipative; then either $R(B-T)=X$ holds for all strongly accretive bounded operators $B$ on $X$, or for no such operators $B$.

Proof. Lemma $2 \mathrm{E}$ is a generalization of the fact that the deficiency $\beta(\lambda-T)$ is constant in the right half plane for dissipative $T$, and can be similarly shown; a semigroup proof is as follows. If $R\left(B_{1}-T\right)=X$, then $R\left[\delta-\left(T-\left(B_{1}-\delta\right)\right)\right]=X$ for $\delta<m_{B_{1}}$, so that by Theorem $2 \mathrm{~A}$ one has $A=T-\left(B_{1}-\delta\right) \in G(1,0)$; for $\varepsilon<m_{B_{2}}$ one has the (dissipative) $T-\left(B_{2}-\varepsilon\right)=A+\left(B_{1}-\delta-B_{2}+\varepsilon\right)$ in $G(1,0)$ by Theorem $2 \mathrm{C}$, so that $R\left[B_{2}-T\right]=R\left[\varepsilon-\left(T-\left(B_{2}-\varepsilon\right)\right)\right]=X$.

As seen in the next section (Proposition 3.1), one has a simple proof of Theorem $2 \mathrm{~B}(\mathrm{i})$ if one uses Theorem $2 \mathrm{C}$, where it is assumed only that $A+B$ is dissipative, rather than using (as was done in the 
original proof of Theorem $2 \mathrm{~B}(\mathrm{i})$ in [8]) the additive result of [7], where it was assumed that $A$ and $B$ are separately dissipative. Lemma $2 \mathrm{E}$ offers another simple proof of Theorem $2 \mathrm{~B}(\mathrm{i})$ as follows; for dissipative $B A$ and bounded strongly accretive $B, R[I-B A]=R[B-B A]=$ $B R[I-A]=X$.

While this paper was in progress, several results concerning additive perturbation of the nonlinear versions of $G(1,0)$ have been obtained; let us mention in particular the paper Crandall and Pazy [2] where Theorem $2 \mathrm{C}$ is generalized to nonlinear $A$ and $B$. For further references to additive perturbation of nonlinear equations of evolution, let us simply refer to the bibliography listed in the recent paper [1]; also in the latter paper the multiplicative perturbation theory is extended to the nonlinear case.

3. Unbounded multiplicative perturbation. One immediately has the following unbounded version of Theorem $2 \mathrm{~B}(\mathrm{i})$.

Proposition 3.1. Let $A \in G(1,0), B A$ dissipative,

$$
\exists \tau>0 \ni\|(\tau B-I) A x\| \leqq a\|x\|+b\|A x\|, \forall x \in D(A), b<1 .
$$

Then $B A \in G(1,0)$.

Proof. Using the decomposition $\tau B A=A+(\tau B-I) A$, we see that $(\tau B-I) A$ is an acceptable additive perturbation via Theorem $2 \mathrm{C}$.

The assumptions of Proposition 3.1 imply that $D[(\tau B-I) A]=$ $D(A)$. In fact one has the general situation

$$
D[(\tau B-I) A]=D(A) \Leftrightarrow R(A) \subset D(B) \Leftrightarrow D(B A)=D(A),
$$

which we mention at this point only to emphasize that domain considerations play an elementary but intrinsic role in determining when decompositions which were automatically valid in the bounded (everywhere defined) case can still be performed. Also, these domain requirements serve to indicate what kind of $B$ can be permitted; for example, according to (3.1), in Proposition 3.1 in those instances (which occur frequently) when $R(A)=X$ one is permitted from among the class of closed operators $B$ only the bounded ones.

Let us recall that for bounded strongly accretive $B$ one knows (see [10]) that $\|\tau B-I\|<1$ for all sufficiently small positive $\tau$, so that Proposition 3.1 extends Theorem $2 \mathrm{~B}(\mathrm{i})$; in this connection let us remark that although clearly (for $\tau>0$ ) $B A \in G(1,0) \Leftrightarrow \tau B A \in G(1,0)$, we include the $\tau$ parameter in our hypotheses since for only a certain 
interval of $\tau$ values will the perturbation inequalities be satisfied (see $[9,10])$.

We now state a somewhat technical but general result.

THEOREM 3.2. The following four conditions are sufficient for $B A \in G(1,0)$ if $A \in G(1,0)$ and $B A$ is dissipative:

(1) $B A$ is closed and densely defined, and

(2) $B(I-A) D(B A)$ is dense, and

(3) $\exists \tau>0 \ni\|(\tau B-I) x\| \leqq a\|x\|+b\|(I-\tau B A) x\|, \forall x \in D(B A)$, where $b<1-a\left\|(I-\tau B A)^{-1}\right\|$, and

(4) $D(B) \supset D(B A)$; (4) is inherent in (3).

Proof. By (1) and Theorem 2A, justifying the above reduces to showing $R(I-\tau B A)$ dense in $X$. For this purpose the identity

$$
(I-\tau B A)+(\tau B-I)=\tau B(I-A)
$$

is useful; that (3.2) is valid on $D(B A)$ is implicit in (3) due to (4). Conditions (2), (3), and Theorem 2D then guarantee that

$$
\beta(I-\tau B A)=\beta\left(\left.B(I-A)\right|_{D(B A)}\right)=0,
$$

so that $R(I-\tau B A)=X$ (the closed range appearing because $I-\tau B A$ is closed and has a bounded inverse).

Let us now examine the conditions (1), (2), (3), (4) under which Theorem 3.2 applies.

If (2) is replaced by the weaker $\left(2^{\prime}\right): \beta(B)=0$, then it is sufficient to replace (4) by either of the stronger conditions: (4') $R((I-$ $A)(D(B A)) \supset D(B) \supset D(B A)$, or $\left(4^{\prime \prime}\right) D(B) \supset D(B A) \supset D(B(I-A))$, both of which imply that in fact $\left(4^{\prime \prime \prime}\right): D(B(I-A))=D(B A)$; or more particularly, by $\left(4^{\prime \prime \prime \prime}\right): D(B) \supset[R(A) \cup D(B A)]$, which holds iff $\left(4^{\prime \prime \prime \prime \prime}\right)$ : $D(B) \supset[R(A) \cup D(A)]$. The latter condition, although mathematically the most convenient class of $B$ if both additive and multiplicative perturbations are to be simultaneously involved (as they are in our decomposition arguments), is highly restrictive, and relates to the following type of situation: if $A$ takes derivatives, $B$ cannot take derivatives. On the other hand, the less restrictive (4) could arise in cases such as those in which both $A$ and $B$ differentiate.

As special cases of (3) due to the dissipativeness of $B A$, either $\left(3^{\prime}\right): b<1-a$, or $\left(3^{\prime \prime}\right): b<1, a=0$, are sufficient; the latter condition is equivalent to $\left(3^{\prime \prime \prime}\right):\left\|(I-\tau B)(I-\tau B A)^{-1}\right\|<1$.

Turning now (1), we see that the "natural" left-multiplicative assumption $D(B) \supset R(A)$ mentioned above (in (3.1)) is clearly sufficient to guarantee $D(B A)$ dense. If that is not the case (e.g., $B$ and $A$ both differential operators) but $A$ is such that $R(A)$ is closed and 
$\beta(A)<\infty$, then (see [6]) $D(B A)$ is dense if $D(B)$ is dense. If $\beta(A)=\infty$ but $A^{-1}$ is bounded, again (for densely defined $B$ ) $D(B A)$ is dense. Concerning when $B A$ is closed, one has by the Fredholm Theory that $B A$ will be closed if $B$ is closed, $R(B)$ is closed, and $\alpha(B) \equiv \operatorname{dim}$ $N(B)<\infty$. To determine when $B A$ is closed from domain considerations alone, one can verify (assuming $D(B) \supset D(B A)$ ) that $B(I-A)=$ $B-B A \Leftrightarrow D(B(I-A))=D(B A)$ i.e., (4"') is satisfied; in that case it may be seen that (3) then implies that

$$
B A \text { closed } \Leftrightarrow I-B A \text { closed } \Leftrightarrow B(I-A) \text { closed . }
$$

This is somewhat advantageous because it is easier in principle to conlcude that $B(I-A)$ is closed rather than that $B A$ is closed, since $I-A$ is a better (invertible) operator than $A$. To continue, assuming for the moment that $R(B)$ is closed, than $R(B(I-A))$ is closed and thus (using (3) and the dissipativeness of $B A$ to show $B(I-A)$ to have a bounded inverse) $B A$ is closed by (3.3). That this is true is of some interest in view of the fact that there exist $B$ closed, $R(B)$ closed, $A$ closed, $R(A)$ closed, $\alpha(A)<\infty, \beta(A)<\infty$, but $B A$ not closed. Under the (4"') assumption made above it also follows that $D(B A)$ is dense whenever $D(B)$ is dense due to the invertibility of $I-A$.

The arguments for the following special instances of Theorem 3.2 are contained in the above discussion.

Corollary 3.3. Let $A \in G(1,0), B A$ dissipative, (3) holding, $D(B)$ dense, $R(B)=X$. Then $B A \in G(1,0)$ under either of the following domain conditions:

(a) $D(B(I-A))=D(B A)$.

(b) $D(B) \supset R(A)$.

Corollary 3.4. Let $A \in G(1,0), B A$ dissipative, $B$ densely defined and invertible, $D(B) \supset D(B A) \supset D(B(I-A))$, and

$$
\exists \tau>0 \ni\left\|(\tau B-I)(I-\tau B A)^{-1}\right\|<1 .
$$

Then $B A \in G(1,0)$.

In a similar way extensions of Theorem $2 \mathrm{~B}$ (ii) to unbounded left multipliers $B$ may be obtained. Since for nonaccretive $B$ this case does not seem to occur as frequently (see [9]), we omit the proof (which is similar to the above) of the following.

Proposition 3.5. Let $A \in G(1,0), B A$ closed, densely defined, and dissipative, $R(B)=X, D(B(I-A))=D(B A)$. If 


$$
\exists \tau>0 \ni\|(\tau B-I) x\| \leqq a\|x\|+b\|\tau B(I-A) x\|,
$$

$\forall x \in D(B A), b<1-a\left\|(\tau B(I-A))^{-1}\right\|$, then $B A \in G(1,0)$.

We remark that one may obtain an alternative to Proposition 3.1 by using the decomposition $I-\tau B A=(I-\tau A)-\tau(B-I) A$, which is always valid and holds on $D(B A)$, as follows. If $A \in G(1,0), D(B A)=$ $D(A), B A$ dissipative, and either of the following conditions (i) or (ii) holds on $D(A)$, then $B A \in G(1,0)$ :

(i) $\tau\|(B-I) A x\| \leqq a\|x\|+b\|(I-\tau B A) x\|$, $b<1-a\left\|(I-\tau B A)^{-1}\right\|$, or

(ii) $\tau\|(B-I) A x\| \leqq a\|x\|+b \| I-\tau A) x\|, b<1-a\|(I-\tau A)^{-1} \|$, for some $\tau>0$. The demonstration consists of noting, in both cases, that $\beta(I-\tau B A)=\beta(I-\tau A)=0, D(B A)$ is dense, and all things needed closed are closed.

We now consider right multiplicative perturbation of $A \in G(1,0)$, using the same type of decompositions that were used for the left multiplicative questions.

Proposition 3.6. Let $A \in G(1,0)$ and $B$ satisfy $\|A(I-\tau B) x\| \leqq$ $a\|x\|+b\|A x\|, b<1, \quad$ some $\tau>0, \forall x \in D(A), D(A B) \supset D(A) ;$ then $A B \in G(1,0)$ if $A B$ is dissipative.

Proof. Since $A(I-B) \supset A-A B$, we note that here $D(B) \supset$ $D(A B) \supset D(A)$. By Theorem 2C, $T=A-A(I-\tau B) \in G(1,0)$ if it is dissipative, and that is the case because $D(T)=D(A)$ and $A B \supset T$. It remains to show that $A B=T$, but this is automatic since it is easily verified that any operator $T \in G(1,0)$ is necessarily maximal dissipative.

Theorem 3.7. Let $A \in G(1,0), A B$ densely defined and dissipative, $R[(A-I) B]=X$. Then $A B \in G(1,0)$ under either of the following conditions:

(i) $\|(\tau B-I) x\| \leqq a\|x\|+b\|(I-\tau A B) x\|, b<1-a\left\|(I-\tau A B)^{-1}\right\|$, $\forall x \in D(A B)$;

(ii) $\quad\|(\tau B-I) x\| \leqq a\|x\|+b \tau\|(A-I) B x\|, b<1-a\left\|((A-I) \tau B)^{-1}\right\|$, $\forall x \in D(A B)$; some $\tau>0$.

Proof. The decomposition $(I-\tau A B)=(I-\tau B)-\tau(A-I) B$ holds identically on $D(A B)$, and thus by Theorem 2D $\beta(I-\tau A B)=$ $\beta((A-I) B)=0$ in either of cases (i) and (ii). Since $R[(A-I) B]$ is closed and $(A-I) B$ is invertible in case (ii), $(A-I) B$ and therefore $I-\tau A B$ are closed, and since the latter also has a bounded inverse, $R[I-\tau A B]=X$ and $A B \in G(1,0)$ in case (ii). Due to the stability of bounded inverses, the inequality in (i) implies that $(A-I) B$ has 
a bounded inverse, and is thus closed, so that $I-\tau A B$, and thus $R[I-\tau A B]$, is closed.

Since right multiplication was not considered in the previous multiplicative perturbation papers $[8,9]$, we now isolate the special case in which $B$ is bounded.

Corollary 3.8. Let $A \in G(1,0), B$ bounded, everywhere defined, and strongly accretive. Then if $A B$ is dissipative, $A B \in G(1,0)$.

Proof. Since there exists $\tau>0$ such that $\|\tau B-I\|<1$, condition (i) of Theorem 3.7 is met; $B$ is invertible so $R[(A-I) B]=X$ and $D(A B)=D[(A-I) B]$ is dense.

A version of Corollary 3.8 with condition (ii) of Theorem $2 \mathrm{~B}$ may be similarly justified.

As a final alternative, we remark that under the following conditions $A B \in G(1,0): A \in G(1,0), A B$ dissipative and densely defined, $\exists \tau>0 \ni$ either of the following holds:

(i) $\tau\|A(I-B) x\| \leqq a\|x\|+b\|(I-\tau A B) x\|, b<1-a\left\|(I-\tau A B)^{-1}\right\|$, or

(ii) $\tau\|A(I-B) x\| \leqq a\|x\|+b\|(I-\tau A) x\|, b<1-a\left\|(I-\tau A)^{-1}\right\|$. The demonstration, the details of which are similar to the others in this paper, utilizes the decomposition $I-\tau A B=(I-\tau A)+\tau A(I-B)$, which holds on $D(A B)$.

One can combine left and right multiplication, as was done in [13], where the setting was a (real) Banach lattice and the negativity condition is one of (weak) dispersiveness rather than dissipativeness.

Theorem 3.9. Let $A \in G(1,0)$, let $D\left(B_{2}\right)=R\left(B_{2}\right)=X$, let $D\left(B_{1}\right)$ be dense and either: (i) $B_{1}^{-1}$ bounded and $R\left(B_{1}\right) \supset D(A)$; or (ii) $B_{1}$ closed and $R\left(B_{1}\right)=X$. Suppose $B_{2} B_{1}$ is bounded and strongly accretive; then $B_{2} A B_{1} \in G(1,0)$ iff it is dissipative.

Proof. Similar to [13, Thm. 3.3], verifying that dissipativeness possesses analogously those properties of dispersiveness needed in the demonstration in [13].

4. Alternate methods and applications of the previous results. In the previous section we utilized operator decompositions and the application of index theory to conclude that the multiplicatively perturbed operator remained in $G(1,0)$; in so doing we were considering the general case in which the operators $B$ and $A$ were not necessarily commutative, invertible, etc.. In the present section we discuss some alternate methods, some applications of the results of $\S 3$, and also we state (Theorem 4.5) when a polynomial in a generator is itself a generator, a result which in turn will be applicable to a number of 
concrete situations (e.g., problems involving higher order differential operators).

Even when the perturbed operator is no longer dissipative one can sometimes prove that it generates a $C_{0}$ semigroup, not necessarily of contractions. We give a result of that type below. Let $G(M, 0)$ denote the infinitesimal generators of $C_{0}$ semigroups $T(t)$ satisfying $\|T(t)\| \leqq M$.

THEOREM 4.1. Let $A \in G(1,0) ; B$ bounded, invertible, and everywhere defined, $B^{2}$ strongly accretive, $B^{-1} A B^{-1}$ dissipative; then $B^{-2} A \epsilon$ $G(M, 0)$ for some $M \geqq 1$.

Proof. Let $X_{1}$ be $X$ renormed by $\|x\|_{1}=\|B x\|$, with semi-inner product $[x, y]_{1}=\left[B^{-1} x, B^{-1} y\right]$ corresponding to the particular semi-inner product for which $B^{-1} A B^{-1}$ is dissipative. Then $\left[B^{-2} A x, x\right]_{1}=$ $\left[B^{-1} A B^{-1}(B x),(B x)\right]$ so that $B^{-2} A$ is dissipative in $X_{1}$, and by Lemma $2 \mathrm{E}$ one has $R\left(I-B^{-2} A\right)=R\left(B^{-2}\left(B^{2}-A\right)\right)=R\left(B^{2}-A\right)=R(T-A)=X_{1}$. Thus $B^{-2} A \in G(1,0)$ in $X_{1}$, so that $B^{-2} A \in G(M, 0)$ for some $M$.

A class of examples of Theorem 4.1 is provided by any $A \in G(1,0)$ in a Hilbert space; then $C A \in G(M, 0)$ for any strongly positive bounded self-adjoint operator $C$, since $B=C^{-1 / 2}$ satisfies the conditions of Theorem 4.1.

In order to illustrate how one can apply the criteria of $\S 3$, let us establish the following lemma, which is certainly known.

Lemma 4.2. Let $A \in G(1,0)$; then $A$ has a bounded inverse iff $R(A)=X$; in particular, $A$ is invertible if $\theta(A)<0$.

Proof. This follows, for example, immediately from the ergodic limit theorem [16, Thm. 18.6.2] which states that $X=\overline{R(A)} \oplus N(A)$.

Now let us consider Corollary 3.4 and let us suppose that $A \in G(1,0)$, $B A$ dissipative, $B$ and $A$ invertible, $D(B) \supset D(B A) \supset D(B(I-A)), A$ commuting with $B$ (i.e., $B A \subset A B$ ). Then for any $x \in D(B A), x=B^{-1} y$ for some $y \in X$, so that for large enough $\tau>0$,

$$
\frac{\|(I-B) x\|}{\|(I-\tau B A) x\|}=\frac{\left\|\left(I-B^{-1}\right) y\right\|}{\left\|B^{-1} y-\tau A y\right\|} \leqq \frac{1+\left\|B^{-1}\right\|}{\tau\left\|A^{-1}\right\|^{-1}-\left\|B^{-1}\right\|}<1,
$$

which is the relative boundedness condition $\left(3^{\prime \prime \prime}\right)$ required in Corollary 3.4; thus $B A \in G(1,0)$.

In particular, let $A \in G(1,0)$ be invertible, and let $B=-A$; then if $-A^{2}$ is dissipative, it is in $G(1,0)$. The accretiveness of $A^{2}$ can 
often be verified directly in specific examples (e.g., where $A$ is a second order differential operator with zero boundary conditions). If $-A$ is a bounded strongly accretive operator on a Hilbert space one can deduce from extensions of the results of [7] that $\cos (-A)>2^{-1 / 2}$ is sufficient for $A^{2}$ to be accretive.

Our intent in this paper has been to perturb $A \in G(1,0)$; however, one can sometimes obtain perturbed results without $A$ a generator to begin with, e.g., as follows.

Proposition 4.3. Let $A$ be invertible; then $-A^{2} \in G(1,0)$ if it is dissipative.

Proof. For small $\delta^{2}, R\left(\delta^{2}-\left(-A^{2}\right)\right)=R((\delta i+A)(-\delta i+A))=X$.

An extension of the above proposition to unbounded right multiplication is the following.

Proposition 4.4. Let $A$ and $B$ be invertible, $A B$ dissipative and densely defined; then $A B \in G(1,0)$.

Proof. For small $\delta, \delta B^{-1}-A$ is invertible so that $R(\delta-A B)=$ $R\left(\delta B^{-1}-A\right)=X$.

Let us now give a rather general theorem covering polynomials $p(A)$ of arbitrary order, without $A$ necessarily invertible, etc.

Theorem 4.5. Let $A \in G(1,0), p(A)$ dissipative, in a complex Banach space. Suppose there exists $\mu, \operatorname{Re} \mu>0$, such that the zeros $\lambda_{i}$ of $q=\mu-p$ all lie in the resolvent $\rho(A)$. The $p(A) \in G(1,0)$.

Proof. We use the index notation for convenience; since the zeros $\lambda_{i}$ of $q=\mu-p$ are in the resolvent set $\rho(A)$, and since $p(A)$ is closed, densely defined, and $\left.\kappa(\mu-p(A))=\sum_{i=1}^{n} \kappa\left(\lambda_{i}-A\right)\right)=0$, by the dissipativeness of $p(A)$ and $\operatorname{Re} \mu>0$ one has $\beta(\mu-p(A))=-\kappa(\mu-p(A))=0$ and thus $\mu \in \rho(p(A))$. It can be verified that $\beta(\lambda-p(A))$ is constant in the right half (open) plane, so $p(A) \in G(1,0)$. Actually it would clearly suffice in Theorem 4.5 to know that the $\lambda_{i}$ are in the Fredholm resolvent set (of index zero).

A little experimentation reveals that Theorem 4.5, due to the freedom of choice of $\mu$, has wide applicability except in the case that the spectrum of $A$ is the whole left half-plane.

It has come to our attention that other investigators have recently obtained results for when $p(A)$ is a generator, from different viewpoints. In papers by Hersh and Griego $[14,15]$, it is assumed that 
$A$ is a group generator, and then, using Fourier transform techniques, it is shown that certain higher order parabolic and hyperbolic Cauchy problems are solvable. In Strang [23], also by Fourier transform methods, followed by an analysis of the numerical ranges of the transformed system, it is shown that operators which are parabolic in the sense of Petrowsky generate holomorphic semigroups.

As an example of multiplicative perturbation in probability questions, let $P(t, x, d y)$ be a temporally homogeneous transition probability for a Markov process, so that $P$ induces (e.g., see [25]) a generator $A \in G(1,0)$ of a contraction semigroup $\left(T_{t} f\right)(x)=\int_{0}^{\infty} P(t, x, d y) f(y)$ on $X=B(0, \infty)$. Letting $w$ be the Markov random function corresponding to $P$, let $B$ be the operator given by multiplication by $p^{-1}$, where $p \in X$ is a strongly positive function; then $P\left(\left[\int_{0}^{t} p(w(s)) d s\right]^{-1}, x, d y\right)$ corresponds to a Markov process with semigroup generator $B A$, and $B$ corresponds to a random time change. For further references and discussion of this application, see [24].

The papers [3,4] were motivated by application of multiplicative perturbation to differential equations, with $B$ a bounded multiplication operator. As a simple example of an unbounded multiplication operator in that type of situation, let $X$ be $C[0,1]$ functions vanishing at 0 and $1, A u=u^{\prime \prime}, D(A)=\left\{u \in X \ni u^{\prime \prime} \in X\right\}$, semi-inner product $[u, v]=$ $u\left(x_{0}\right) \overline{v\left(x_{0}\right)}$, where $v\left(x_{0}\right)=\|v\|=\sup |v(x)|$, and let $B$ be given by multiplication by $p$, where $p \in C(0,1)$, possibly unbounded as $x \rightarrow 0,1$, but strongly positive, i.e., $p(x) \geqq m>0$. Then $B A-\varepsilon B \in G(1,0)$ for any positive $\varepsilon$, by Proposition 4.4. Other examples can be similarly constructed with $B$ a strongly positive multiplication operator and $A$ a differential operator of local type in a Banach space of continuous functions, but we shall not go into the details here.

\section{REFERENCES}

1. B. Calvert and K. Gustafson, Multiplicative perturbation of nonlinear m-accretive operators, J. Funct. Anal. 10 (1972), 149-158.

2. M. G. Crandall and A. Pazy, Semigroups of nonlinear contractions and dissipative sets, J. Funct. Anal., 3 (1969), 376-418.

3. J. R. Dorroh, Contraction semigroups in a fuction space, Pacific J. Math., 19 (1966), $35-38$.

4. - Some properties of a partial differential operator, Illinois J. Math., 11 (1967), 177-188.

5. J. R. Giles, Classes of semi-inner product spaces, Trans. Amer. Math. Soc., 129 (1967), 436-446.

6. S. Goldberg, Unbounded Linear Operators, McGraw-Hill, New York (1966).

7. K. Gustafson, A perturbation lemma, Bull. Amer. Math. Soc., 72 (1966), 334-338.

8. - A note on left multiplication of semigroup generators, Pacific J. Math., 24 (1968), 463-465.

9. - Positive (noncommuting) operator products and semigroups, Math. Zeit., 
105 (1968), 160-172.

10. The angle of an operator and positive operator products, Bull. Amer. Math. Soc., 74 (1968), 488-492.

11. Doubling perturbation sizes and preservation of operator indices in normed linear spaces, Proc. Cambridge Philos. Soc., 66 (1969), 281-294.

12. K. Gustafson and B. Zwahlen, On the cosine of unbounded operators, Acta Scientiarum Mathematicarum, 30 (1969), 33-34.

13. K. Gustafson and Ken-iti Sato, Some perturbation theorems for nonnegative contraction semigroups, J. Math. Soc. Japan, 21 (1969), 200-204.

14. R. Hersh and R. Griego, Random evolutions, markov chains, and systems of partial differential equations, Proc. Nat. Acad. Sci., U.S.A., 62 (1969), 305-308.

15. - Direct solution of general one-dimensional linear parabolic equations via an abstract Plancherel formula, Proc. Nat. Acad. Sci., U.S.A., 63 (1969), 648-654. 16. E. Hille and R. S. Phillips, Functional analysis and semigroups, Amer. Math. Soc. Colloq. Publ., 31, revised edition (1957), Providence, R. I.

17. T. Kato, Some mapping theorems for the numerical range, Proc. Jap. Acad., 41 (1965), 652-655.

18. - Perturbation theory for linear operators, Springer-Verlag, Berlin (1956).

19. G. Lumer, Semi-inner product spaces, Trans. Amer. Math. Soc., 100 (1961), 29-43.

20. - On the isometries of reflexive Orlicz spaces, Ann. Inst. Fourier, 13 (1963), 99-109.

21. G. Lumer and R. S. Phillips, Dissipative operators in a Banach space, Pacific J. Math., 11 (1961), 679-698.

22. D. K. Rao, Perturbed Numerical Range and Initial Value Problems, thesis, Univ. of Colorado, 1971.

23. G. Strang, On numerical ranges and holomorphic semigroups, J. Analyse Math., 22 (1969), 299-318.

24. C. Widger, Multiplicative Perturbation of Generators of Semigroups of Operators, thesis, Univ. of Washington, 1970.

25. K. Yosida, Functional analysis, Springer-Verlag, Berlin (1966).

Received March 16, 1970, and in revised form June 23, 1971. The first author was partially supported by NSF Grant GP-15239 and the second author was partially supported by NSF Grant GP-22727.

University of Colorado and Ecole Polytechnique Federale lausanne AND

UNIVERSity OF WAShington aND UNIVERSity of Paris 


\title{
PACIFIC JOURNAL OF MATHEMATICS
}

\author{
EDITORS
}

H. SAMELSON

Stanford University

Stanford, California 94305

C. R. HOBBY

University of Washington

Seattle, Washington 98105

\section{J. DugundJI}

Department of Mathematics

University of Southern California

Los Angeles, California 90007

RICHARD ARENS

University of California

Los Angeles, California 90024

\section{ASSOCIATE EDITORS}
E. F. BeCKeNBACH
B. H. NeUmanN
F. WoLF
K. YoSHIDA

\section{SUPPORTING INSTITUTIONS}

\author{
UNIVERSITY OF BRITISH COLUMBIA \\ CALIFORNIA INSTITUTE OF TECHNOLOGY \\ UNIVERSITY OF CALIFORNIA \\ MONTANA STATE UNIVERSITY \\ UNIVERSITY OF NEVADA \\ NEW MEXICO STATE UNIVERSITY \\ OREGON STATE UNIVERSITY \\ UNIVERSITY OF OREGON \\ OSAKA UNIVERSITY
}

\author{
UNIVERSITY OF SOUTHERN CALIFORNIA \\ STANFORD UNIVERSITY \\ UNIVERSITY OF TOKYO \\ UNIVERSITY OF UTAH \\ WASHINGTON STATE UNIVERSITY \\ UNIVERSITY OF WASHINGTON

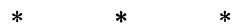 \\ AMERICAN MATHEMATICAL SOCIETY \\ NAVAL WEAPONS CENTER
}

The Supporting Institutions listed above contribute to the cost of publication of this Journal, but they are not owners or publishers and have no responsibility for its content or policies.

Mathematical papers intended for publication in the Pacific Journal of Mathematics should be in typed form or offset-reproduced, (not dittoed), double spaced with large margins. Underline Greek letters in red, German in green, and script in blue. The first paragraph or two must be capable of being used separately as a synopsis of the entire paper. The editorial "we" must not be used in the synopsis, and items of the bibliography should not be cited there unless absolutely necessary, in which case they must be identified by author and Journal, rather than by item number. Manuscripts, in duplicate if possible, may be sent to any one of the four editors. Please classify according to the scheme of Math. Rev. Index to Vol. 39. All other communications to the editors should be addressed to the managing editor, Richard Arens, University of California, Los Angeles, California, 90024.

50 reprints are provided free for each article; additional copies may be obtained at cost in multiples of 50 .

The Pacific Journal of Mathematics is published monthly. Effective with Volume 16 the price per volume (3 numbers) is $\$ 8.00$; single issues, $\$ 3.00$. Special price for current issues to individual faculty members of supporting institutions and to individual members of the American Mathematical Society: $\$ 4.00$ per volume; single issues $\$ 1.50$. Back numbers are available.

Subscriptions, orders for back numbers, and changes of address should be sent to Pacific Journal of Mathematics, 103 Highland Boulevard, Berkeley, California, 94708.

PUBLISHED BY PACIFIC JOURNAL OF MATHEMATICS, A NON-PROFIT CORPORATION

Printed at Kokusai Bunken Insatsusha (International Academic Printing Co., Ltd.), 270, 3-chome Totsuka-cho, Shinjuku-ku, Tokyo 160, Japan. 


\section{Pacific Journal of Mathematics}

\section{Vol. 41, No. $3 \quad$ BadMonth, 1972}

George E. Andrews, Two theorems of Gauss and allied identities proved arithmetically.................................. 563

Stefan Bergman, On pseudo-conformal mappings of circular domains . . . . 579

Beverly L. Brechner, On the non-monotony of dimension ............ 587

Richard Anthony Brualdi and John H. Mason, Transversal matroids and Hall's theorem .................................... 601

Philip Throop Church and James Timourian, Differentiable maps with

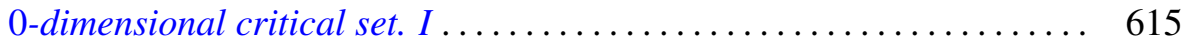

John H. E. Cohn, Squares in some recurrent sequences ............. 631

Robert S. Cunningham, Edgar Andrews Rutter and Darrell R. Turnidge, Rings of quotients of endomorphism rings of projective modules ......

Eldon Dyer and S. Eilenberg, An adjunction theorem for locally equiconnected spaces................................... 669

Michael W. Evans, On commutative P. P. rings................. 687

Ronald Lewis Graham, Hans Sylvain Witsenhausen and Hans Zassenhaus, On tightest packings in the Minkowski plane ..................

Stanley P. Gudder, Partial algebraic structures associated with

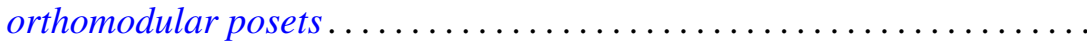

Karl Edwin Gustafson and Gunter Lumer, Multiplicative perturbation of

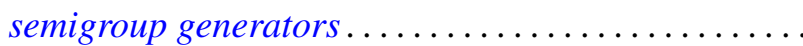

Kurt Kreith and Curtis Clyde Travis, Jr., Oscillation criteria for selfadjoint elliptic equations...

Lawrence Louis Larmore, Twisted cohomology theories and the single obstruction to lifting...........................

Jorge Martinez, Tensor products of partially ordered groups . . .

Robert Alan Morris, The inflation-restriction theorem for Amitsur cohomology ...

Leo Sario and Cecilia Wang, The class of $(p, q)$-biharmonic functions ...

Manda Butchi Suryanarayana, On multidimensional integral equations of

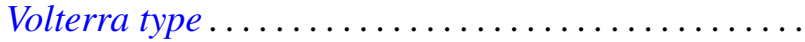

